\title{
Diseño y aplicación de un Índice de Calidad de Vida en áreas urbanas: el caso de la Cuenca Matanza Riachuelo
}

\section{Design and application of a Quality of Life Index in urban areas: the case of the Matanza- Riachuelo basin}

María Laura Henry

Doctora en Ciencias Sociales. Facultad de Humanidades y Ciencias de la Educación. Universidad Nacional de La Plata. Calle 51 e/ 124 y 125 , (1925) Ensenada, Buenos Aires, Argentina, mlaurahenry@gmail.com, ORCID https://orcid.org/0000-0002-2869-8506

Marcos Cipponeri

Ingeniero Hidráulico e Ingeniero Civil. Facultad de Ingeniería. Universidad Nacional de La Plata. Calle 47 N²00, (1900) La Plata, Buenos Aires, Argentina, mcipponeri@ing.unlp.edu.ar, ORCID https://orcid.org/0000-0002-9533-4366

Valentina Bonifacio

Magister en Economía. Fondo Monetario Internacional. 1900 Pennsylvania Ave, Washington, Distrito de Columbia, Estados Unidos, valentina.bonifacio28@gmail.com, ORCID https://orcid.org/0000-0002-6811-3437

Mónica Laura Salvioli

Licenciada en Biología con orientación Ecología. Especialista en Ambiente y Patología Ambiental. Universidad Nacional de La Plata. Facultad de Ingeniería. UIDET Gestión Ambiental. Calle 47 N²00, (1900) La Plata, Buenos Aires, Argentina, msalvioli@ing.unlp.edu.ar, ORCID https://orcid.org/0000-0002-4104-1701

\section{Guillermo Larrivey}

Ingeniero Hidráulico e Ingeniero Civil. Unidad de Investigación, Desarrollo, Extensión y Transferencia UIDET. Facultad de Ingeniería. Universidad Nacional de La Plata. Calle $47 N^{\circ}$ 200, (1900) La Plata, Buenos Aires, Argentina, glarrivey@ing.unlp.edu.ar, ORCID https://orcid.org/0000-0002-3481-3152

Verónica Guerrero Borges

Magister en Ciencias del Territorio. Facultad de Ciencias Naturales y Museo. Facultad de Ingeniería. Universidad Nacional de La Plata. Av. 1 750, (1900) La Plata, Buenos Aires, Argentina, vgborges@gmail.com, ORCID https://orcid.org/0000-0002-0231-9918

Recibido: 28 de febrero 2020 || Aprobado: 16 de junio 2020

Resumen

Este trabajo tiene como objetivo exponer el diseño de un Índice de Calidad de Vida (ICV) adaptado a la realidad socioambiental de la Cuenca Matanza Riachuelo (localizada en Argentina), describir sus principales aspectos metodológicos y mostrar los resultados obtenidos a partir de su aplicación en el año 2016, así como sus potenciales usos prácticos. En el artículo se describen los pasos realizados para desarrollar el ICV: diagnóstico socioambiental de la cuenca, amplio análisis de la bibliografía existente, elaboración de un marco conceptual para precisar en qué consiste la calidad de vida para la cuenca, definición y selección de las dimensiones e indicadores a incluir en el ICV, y, por último, mapeo de los mismos a través de un Sistema de Información Geográfica (SIG). Los resultados muestran la existencia de heterogeneidades significativas en el territorio bajo estudio. Asimismo, se vislumbra que las características intrínsecas de la cuenca son un factor significativo para comprender el patrón de distribución del ICV, lo cual muestra la importancia de tomar las cuencas como unidades de análisis y de gestión.

Palabras clave: Calidad de vida; Cuencas urbanas; Indicadores ambientales; Saneamiento

Cita sugerida: Henry, M.L.; Cipponeri, M.; Bonifacio, V.; Salvioli, M.L.; Larrivey, G. y Guerrero Borges, V. (2020). Diseño y aplicación de un Índice de Calidad de Vida en áreas urbanas: el caso de la cuenca Matanza-Riachuelo. Estudios Socioterritoriales. Revista de Geografía, (28), 055. DOI: https://doi.org/10.37838/unicen/est.28-055 
Abstract

This article aims to expose the design of a Quality of Life Index (QLI) adapted to the socio-environmental reality of the Matanza Riachuelo Basin (located in Argentina), describe its main methodological aspects and show the results obtained from its application in the year 2016, as well as its potential practical uses. The text describes the steps taken to develop the QLI: socio-environmental analysis of the basin, extensive examination of the existing bibliography, elaboration of a conceptual framework to specify the quality of life for the basin, definition and selection of the dimensions and indicators included in the ICV, and, finally, the mapping process through a Geographic Information System (GIS). The results show the existence of significant heterogeneities in the territory under study. It also demonstrates that the intrinsic characteristics of the basin are a significant factor in order to understand the distribution pattern of the QLI, which shows the importance of taking the basins as units of analysis and of administration.

Key words: Quality of life; Urban basins; Environmental indicators; Sanitation

\section{INTRODUCCIÓN}

La expresión calidad de vida es frecuentemente utilizada en distintos ámbitos y niveles. Al nivel de las personas y las familias, se la suele invocar como aspiración en términos de conseguir ciertos niveles de bienestar. En el campo de la opinión pública, suele ser utilizada por periodistas, políticos y profesionales de diversas disciplinas para evaluar el estado de situación de la población y como fin primordial a los cuales los esfuerzos públicos y privados deben estar orientados.

En todos los casos, lo que se refleja en el uso del concepto calidad de vida es la preocupación que existe en la actualidad por evaluar el grado en que una sociedad puede brindar a sus miembros distintos medios, recursos y capacidades que les permitan desarrollarse plenamente. En este sentido, es innegable la dimensión ética que despierta la cuestión. Pero simultáneamente, debatir sobre la calidad de vida también genera una serie de interrogantes prácticos, dado que se requieren herramientas (entre otras, un sistema de índices e indicadores) que permitan evaluarla de manera fehaciente y que, posteriormente, orienten e identifiquen las intervenciones necesarias para mejorarla.

Los potenciales usuarios de los indicadores (de calidad de vida y de otros temas) son los funcionarios públicos de distintos niveles, líderes políticos y líderes comunitarios, que hacen uso de los mismos para determinar situaciones y marcar tendencias (presentando unos pocos valores), a diferencia de los datos crudos o procesados utilizados por técnicos, especialistas e investigadores, que poseen un mayor nivel de desagregación (Fasciolo, Puebla, Mendoza y Cifuentes, 2011).

En el marco de estas preocupaciones, este trabajo tiene como objetivo exponer el diseño de un ICV adaptado a la realidad socioambiental de la Cuenca Matanza Riachuelo, describir sus principales aspectos metodológicos y mostrar los resultados obtenidos a partir de su aplicación en el año 2016, así como sus potenciales usos prácticos.

La temática bajo análisis tiene como antecedente un recorrido de tipo conceptual y metodológico realizado por el equipo de investigación de la Universidad Nacional de La Plata (UNLP), en el cual se ha indagado en profundidad tanto el constructo 
teórico de calidad de vida como en las opciones metodológicas para su medición concreta, en el marco de actividades de investigación y transferencia desde el ámbito universitario al de la gestión pública' ${ }^{1}$.

\section{EL ÁMBITO BAJO ESTUDIO}

La Cuenca Matanza Riachuelo tiene una superficie aproximada de $2.200 \mathrm{~km} 2$ y abarca la parte sur de la Ciudad Autónoma de Buenos Aires (CABA) y parte del territorio de 14 partidos de la provincia de Buenos Aires. Está altamente urbanizada, con una población que supera los cuatro millones de habitantes, la cual constituye aproximadamente el $25 \%$ de la población de la provincia de Buenos Aires y el 10\% de la del país. En esta cuenca conviven actividades residenciales, industriales, agrícola/ganaderas, comerciales, entre las principales. Asimismo, es posible encontrar barrios marginales, villas y asentamientos que conviven con barrios residenciales de clase media, barrios cerrados y clubes de campo.

Además, es la cuenca más contaminada del país y en sus cursos de agua son vertidos efluentes industriales con escaso o nulo tratamiento, contaminantes de origen cloacal y grandes cantidades de residuos sólidos. Dada esta situación, en 2004 un grupo de vecinos presentó una demanda contra el Estado Nacional, la provincia de Buenos Aires, la Ciudad Autónoma de Buenos Aires y 44 empresas, reclamando la recomposición del ambiente, la creación de un fondo para financiar el saneamiento de la cuenca y un resarcimiento económico por daños y perjuicios. Posteriormente, la demanda se amplió hacia los 14 municipios bonaerenses, abarcando así la Cuenca Matanza Riachuelo (ACUMAR, 2016). Este fue el origen de la denominada Causa Mendoza (SAIJ, 2008).

El 8 de julio de 2008, la Corte Suprema de Justicia de la Nación dictó un fallo histórico donde se determinó quiénes serían los responsables de llevar adelante las acciones y las obras de saneamiento. Particularmente, se ordenó la creación de un Organismo de Cuenca: la Autoridad de Cuenca Matanza Riachuelo (ACUMAR). Este organismo interjurisdiccional lleva adelante desde hace varios años un programa cuyos objetivos son: 1) la mejora de la calidad de vida de los habitantes de la cuenca; 2) la recomposición del ambiente en todos sus componentes (agua, aire y suelo); y 3) la prevención de daños con suficiente y razonable grado de predicción (ACUMAR, 2016).

En el año 2015, ACUMAR solicitó a la UNLP que desarrolle un Índice de Calidad de Vida (ICV) que permita guiar las acciones estructurales y no estructurales a implementar en la cuenca y a monitorear la evolución de la calidad de vida de sus habitantes. En dicho marco se inició el desarrollo de esta herramienta, lo cual implicó un proceso muy rico en términos conceptuales y metodológicos y que se detalla en los apartados que siguen.

\section{ORÍGENES Y DESARROLLO DE LOS INDICADORES SOBRE CALIDAD DE VIDA}

Adentrarse en el estudio de la calidad de vida implica abordar un campo complejo, compuesto por múltiples temáticas interrelacionadas y donde confluyen distintas disciplinas. Por ello, una reflexión preliminar sobre este campo temático y sobre sus intentos de medición empírica constituye una tarea fundamental que permite comprender mejor su especificidad.

1 Los resultados que se exponen en este artículo fueron desarrollados en el marco del Convenio Específico $\mathrm{N}^{\circ}$ 1 UNLP-ACUMAR "Proyecto para la evaluación de Indicadores de Calidad de Vida e Índices de Calidad de Aguas en la Cuenca Matanza-Riachuelo". Este proyecto fue ejecutado por la UIDET Gestión Ambiental, Departamento de Hidráulica, Facultad de Ingeniería de la Universidad, en los años 2015-2017. 
Una primera observación que debe hacerse respecto del concepto de calidad de vida es que el mismo se ha propuesto como un intento por enriquecer aquellos enfoques tradicionales, que reducían dicha temática a un plano estrictamente económico y monetario. De esta forma, en un principio se postulaba que la medición de los recursos económicos de la población -en tanto medios para la adquisición de ciertos bienes y servicios- constituían una aproximación bastante adecuada a los fines de medir su bienestar y su calidad de vida (Gil Ospina, 2003; Pena Trapero, 2009).

Esta forma de concebir la calidad de vida logró posicionarse como hegemónica durante mucho tiempo y tuvo su correlato a nivel metodológico. En este sentido, la primera herramienta que se propuso para medirla a nivel país fue el Producto Bruto Nacional o Interno (PBI) o, de forma similar, el ingreso per cápita, resultado de dividir el PBI entre el total de la población de un país (Espinosa Ortiz, 2014).

Pero las posteriores elaboraciones conceptuales fueron poniendo en evidencia que medir la calidad de vida solo desde el plano económico puede resultar un enfoque demasiado estrecho, ya que deja por fuera del análisis una serie de aspectos que las personas demandan y necesitan para alcanzar su desarrollo integral (Cobb, 2000; Gil Ospina, 2003; Camargo Mora, 1999; Actis Di Pasquale, 2008).

De esta forma, en las últimas décadas han tomado fuerza enfoques que plantean una reflexión enriquecida sobre la calidad de vida y sobre las formas de sopesar en qué medida una población se halla en condiciones de autorrealizarse en sus potencialidades humanas plenas. Estos enfoques renovadores introducen una mirada multidimensional para la medición del bienestar, donde la integración de diferentes atributos queda sintetizada en un índice, permitiendo realizar una comparación del bienestar entre diferentes sujetos o grupos dentro de una sociedad.

En los últimos años se ha recorrido un camino muy fructífero en la elaboración y estudio de indicadores de calidad de vida. Nuevas dimensiones y variables se han ido adicionando en las propuestas de medición, acompañando el creciente interés de gobiernos, investigadores y ciudadanos por conocer los cambios acaecidos en este plano. A nivel internacional podemos encontrar trabajos de organismos internacionales, de los gobiernos de distintos países y de ámbitos académicos en dicha línea: OECD (2011); Castaño (2008); Ministerio de Planificación Metropolitana (2010); Precedo Ledo, Míguez Iglesias y Orosa González (2012); United Nations (2004).

En nuestro país también es posible encontrar trabajos donde se han desarrollado y aplicado índices orientados a medir el bienestar y la calidad de vida de la población desde un enfoque multidimensional: Velázquez, Longhi, Mikkelsen y Celemín (2013); Leva (2005); Prieto (2013); Discoli et al. (2013); Zulaica y Celemín (2008); Actis Di Pasquale (2015). En estos trabajos citados pueden encontrarse diferencias ligadas a las formas de medición, las fuentes usadas, los ámbitos de análisis, sus enfoques disciplinares y los marcos institucionales de elaboración, entre otros aspectos.

Incluso el Instituto Nacional de Estadística y Censos (INDEC) de Argentina ha reconocido recientemente el valor de este tipo de mediciones y desde el año 2016 ofrece periódicamente un Informe de Indicadores de Condiciones de Vida de los hogares en 31 aglomerados urbanos (INDEC, 2019)2. Por último, es importante referenciar el instrumento interactivo

2 Estos indicadores surgen de la combinación de algunas variables relevadas por la Encuesta Permanente de Hogares $(\mathrm{EPH})$ en 31 aglomerados urbanos. La información presentada responde a distintas dimensiones: características habitacionales, servicios públicos, características del hábitat, régimen de tenencia de la vivienda, cobertura médica y educación. 
online denominado Mapa del Índice de Calidad de Vida (ICV)3 ${ }^{3}$, que permite conocer el nivel de calidad de vida en los más de 52 mil radios censales en los que se divide la Argentina. Al igual que el instrumento que presentamos en este artículo, el mencionado mapa usa como escala de medición los radios censales, pero está compuesto por distintos indicadores y no está diseñado específicamente para cuencas. En este sentido, consideramos que el ICV aquí propuesto puede ser un aporte complementario y original para el fructífero campo de estudio de la calidad de vida en nuestro país.

\section{EL CONCEPTO DE CALIDAD DE VIDA UTILIZADO EN NUESTRO TRABAJO}

Para proceder a medir la calidad de vida se requiere, como paso previo y fundamental, una definición de dicho concepto para así establecer qué áreas caen bajo su órbita y qué aspectos deberán ser relevados empíricamente. Al respecto, aquí se propone una definición que ha sido fruto de un proceso de discusión interdisciplinaria al interior del equipo de investigación. En su elaboración se ha buscado que tenga el grado suficiente de especificidad para delimitar el campo de análisis, pero también que dicha definición tenga un rango de amplitud adecuado como para albergar las múltiples dimensiones y elementos que la constituyen.

Al respecto, se propone entonces la siguiente definición: "la calidad de vida se refiere al grado en que la población, de un territorio específico, logra disponer de una serie de recursos de infraestructura, sanitarios, educativos y ambientales que le permiten satisfacer sus necesidades humanas, decidir sobre sus trayectorias vitales y desarrollarse integralmente en un marco de equidad social".

Como tal, la calidad de vida remite entonces a ciertos estándares de bienestar y de oportunidades, que son objeto de una construcción colectiva por parte de actores, instituciones y organizaciones públicas y privadas.

Otro aspecto conceptual que debe señalarse está referido al mencionado anclaje territorial de la calidad de vida. Al respecto, este trabajo propone la medición de la calidad de vida en las cuencas hidrográficas a partir de las potencialidades analíticas que conlleva este recorte espacial. Siguiendo a Dourojeanni (1994) es posible afirmar que se trata de un territorio que es delimitado por la propia naturaleza, definiendo el área a través de la cual las aguas superficiales convergen hacia un mismo cauce. Los recursos naturales y los habitantes de una cuenca poseen condiciones (físicas, biológicas, económicas, sociales y culturales) compartidas, que le confieren características particulares a cada una. Según Dourojeanni, Jouravlev y Chávez (2002, p. 47) "las cuencas son territorios útiles para lograr metas tangibles de 'desarrollo sustentable' adaptables a las condiciones de cada lugar (compatibilizar niveles de calidad de vida con lo que puede ofrecer el territorio y la organización social y económica)".

En definitiva, cuando es posible definir con precisión una cuenca o subcuenca, se tornan en unidades pertinentes para el análisis de la calidad de vida y, por otro, como unidades de gestión para su mejora. En este último caso, la ejecución de planes, programas y proyectos tendrá mayor efectividad por la mencionada integración que presentan las mismas.

3 Para accederse al mismo y conocer más detalles sobre su diseño, ver el sitio web: https://icv.conicet.gov.ar/ 


\section{LAS DIMENSIONES DE LA CALIDAD DE VIDA}

El ICV utilizado en este trabajo ha sido organizado en cuatro dimensiones y cada una de ellas aborda un aspecto específico de ese conjunto global que ha sido definido como calidad de vida. La selección de estas dimensiones ha sido resultado de sopesar dos cuestiones complementarias. Por un lado, se han tomado en cuenta las características y las problemáticas existentes en la región bajo estudio, ya que un instrumento de este tipo debe ser sensible a las características del territorio bajo análisis y a las realidades que atraviesa su población. Por otro lado, para seleccionar las dimensiones se han tomado como referencia aquellos aspectos donde puedan proyectarse intervenciones factibles y asequibles en el marco de los recursos e instituciones públicas ${ }^{4}$.

Asimismo, cabe indicar que en el ICV se decidió no incluir, de manera directa, la dimensión estrictamente económica (con aspectos referentes al ingreso salarial, a la condición ocupacional, etc.). A diferencia de otros esfuerzos de medición, aquí se considera que una evaluación de la calidad de vida debe centrarse en los resultados reales que generan dichas variables económicas y en las experiencias concretas de vida de la población. En todo caso, estas variables económicas quedarán indirectamente mensuradas a partir de las condiciones en las que viven efectivamente las personas.

Habiendo hecho estas puntualizaciones, a continuación se enuncian las cuatro dimensiones incluidas en el ICV y el enfoque adoptado para cada una de ellas:

a) Dimensión vivienda: la vivienda representa un componente fundamental en el bienestar de la población y es esencial para que sus miembros puedan desarrollar su vida con seguridad y con dignidad y en tal sentido es reconocida en la Ley Provincial $N^{\circ} 14.449$ (SAIJ, 2013a) $)^{5}$. Pero no cualquier vivienda sirve para dichos propósitos, debe reunir una serie de condiciones que la hagan apta para su habitabilidad. Las condiciones seleccionadas como representativas de una vivienda digna han sido: calidad de sus materiales, acceso a gas por red, hacinamiento y certeza en el uso del dominio.

b) Dimensión salud: la salud se considera un bien básico y valioso, ya que permite llevar una vida plena y una existencia prolongada en el tiempo. En este trabajo, la salud es entendida desde un enfoque que estima la necesidad de contemplar los múltiples aspectos que influyen sobre la misma y asimismo, se ha puesto énfasis en el plano de la prevención. Bajo estas pautas, se han seleccionado los siguientes indicadores: existencia de un centro de atención primaria cercano al hogar; acceso simultáneo a agua potable y cloacas a través de redes (servicios sanitarios básicos, SSB), reconocidos como derecho humano por la Ley Provincial № 14.782 (SAlJ, 2013b); y cercanía a basurales a cielo abierto (como aspecto que influye negativamente sobre la salud) ${ }^{6}$.

4 Como se dijo antes, la calidad de vida no se considera el resultado de acciones y recursos individuales, sino que es una construcción colectiva. En este marco, el Estado juega un papel fundamental, ya sea por su acción directa, o por medio del establecimiento de regulaciones que orientan el accionar de los actores públicos y privados.

5 Específicamente en su artículo $3^{\circ}$, indica: “Derecho a la vivienda. Definición. El derecho a una vivienda y a un hábitat digno comporta la satisfacción de las necesidades urbanas y habitacionales de los ciudadanos de la Provincia, especialmente de quienes no logren resolverlas por medio de recursos propios, de forma de favorecer el ejercicio pleno de los derechos fundamentales" (SAIJ, 2013a).

6 En su artículo $2^{\circ}$, se define como saneamiento al "manejo sanitario del agua potable, las aguas residuales y excretas, los residuos sólidos y los hábitos higiénicos". Asimismo, en dicho artículo se indica que, gestionados adecuadamente estos elementos del saneamiento "reducen los riesgos para la salud y previenen los impactos sobre el medio ambiente, cuya finalidad última es la promoción y el mejoramiento de condiciones de vida de la población urbana y rural” (SAIJ, 2013b). 
c) Dimensión educación: la educación es uno de los elementos prioritarios dentro del bienestar social, a tal punto que el derecho a aprender está incluido en nuestra Constitución Nacional en su artículo 14 y está ampliado y reforzado por los derechos referidos a la educación en sus artículos 41 y 42 . Esto se debe a que la educación brinda conocimientos, habilidades y valores necesarios para que los individuos puedan desenvolverse socialmente y desarrollarse integralmente. En este caso los indicadores seleccionados para representar esta dimensión han sido: esperanza de años de escolaridad de la población menor a 25 años y la escolaridad media alcanzada por la población mayor a 25 años de edad. Estos indicadores, han sido tomados del componente educación del Índice de Desarrollo Humano de Naciones Unidas.

d) Dimensión entorno: el uso del espacio urbano se ha vuelto objeto de atención prioritaria para las políticas públicas, donde distintas acciones de ordenamiento buscan contribuir a la calidad de vida de la población. Específicamente, el concepto de entorno hace referencia a aquella porción del ambiente (urbano) que interactúa significativamente con la comunidad en su lugar de residencia, incidiendo en su calidad de vida. La misma Constitución Nacional menciona en su artículo 41: "Todos los habitantes gozan del derecho a un ambiente sano, equilibrado, apto para el desarrollo humano y para que las actividades productivas satisfagan las necesidades presentes sin comprometer las de las generaciones futuras". Se relevaron entonces para el ICV elementos del territorio cercano que pueden tener una incidencia positiva (espacio verde público y transporte público) o que pueden tener un efecto negativo sobre la calidad de vida (cavas, industrias e inundaciones).

\section{Metodología}

Según la definición adoptada, el ICV propuesto mide la calidad de vida de la población urbana en la Cuenca Matanza Riachuelo a nivel de radio censal, la menor unidad geográfica sobre la cual recaba datos el Censo Nacional de Población, Hogares y Viviendas (INDEC, 2010) (fuente que proveyó aproximadamente el 50\% de los datos que componen el ICV). Se consideraron 'urbanos' todos los radios censales con densidad mayor a 10 habitantes por hectárea, asumiendo que la misma es una densidad mínima razonable para llegar con los servicios públicos. Asimismo, esta condición umbral permite abarcar zonas donde la población está efectivamente agrupada ${ }^{7}$ y mantiene la comparabilidad con aquellas zonas más densamente pobladas de la cuenca.

Los indicadores que lo componen se calcularon mediante la utilización de un Sistema de Información Geográfica (SIG) que, a través de algoritmos específicos, combinaron información de población a nivel de radio censal con información que permitiera darle sentido a los mismos, como lo es la ubicación de cavas, de centros de atención primaria, de espacios verdes públicos, de industrias o características propias de la población como la calidad de sus viviendas o los niveles de educación alcanzados, a modo de ejemplo.

Cada indicador establecido representa un porcentaje de población, de un determinado radio censal, que cumple con determinadas condiciones o que es influenciada por factores de su entorno. Dado este diseño, los resultados de cada indicador varían entre 0 y 1 (correspondiendo estos extremos a $0 \%$ y $100 \%$ de la población del radio censal).

7 Según el Indec un radio "urbano" es aquel donde puede verificarse población agrupada, mientras que uno "rural" es aquel donde existe población dispersa únicamente, y donde las viviendas se distribuyen en campo abierto en forma diseminada (INDEC, 2015). 
Al agregar los indicadores correspondientes a cada dimensión podemos obtener un subíndice asociado a la misma, cuyos valores extremos también varían entre 0 y 1 , pero ya no representan un porcentaje de la población del radio censal, sino que es un valor que representa los niveles alcanzados por la dimensión bajo estudio (muestra el mejor o peor estado de dicha dimensión en el territorio). Lo mismo ocurre cuando se agregan los sub-índices de las cuatro dimensiones y se obtiene el ICV.

El diseño de los indicadores se ha realizado de modo que aporten positivamente al ICV. Por ello, en aquellos casos en que los aspectos analizados aportaban negativamente a la calidad de vida (tales como presencia de basurales, de cavas o de industrias), hubo que diseñar los algoritmos para cumplir con la condición mencionada (de aporte positivo al ICV). Esto permitió que las fórmulas de agregación de indicadores o de subíndices no conllevaran términos o factores negativos.

Para la representación gráfica de los indicadores, subíndices e ICV se trabajó con intervalos en los que se vincularon valores numéricos con características cualitativas y con colores (Tabla 1).

Los subíndices vinculados a cada una de las cuatro dimensiones se calculan también, mediante SIG, aplicando las fórmulas indicadas más abajo, lo mismo que el ICV, que combina, sintetiza y simplifica la información que contienen los primeros.

Dado que el ICV pretende capturar el nivel de vida de las personas, la cantidad de población por radio censal es un insumo relevante. Por la cobertura de sus datos, la fuente por excelencia es el censo, siendo el último disponible, el Censo Nacional de Población, Hogares y Viviendas del año 2010 (INDEC, 2010). La ventaja de esta fuente es que se evitan realizar estimaciones sobre muestras representativas, operando directamente sobre los datos de la población. Por otro lado, la desventaja de permanecer fiel a esta fuente radica en su periodicidad. Los censos se realizan cada diez años, siendo extenso el espacio temporal que media entre los mismos, donde no solo puede cambiar el tamaño de la población, sino también su distribución y características socioeconómicas en el territorio. Por este motivo, es que se decidió acudir a otras fuentes complementarias para completar la información. En este sentido, se incorporaron otras fuentes secundarias de organismos estatales, datos provistos por informantes claves, imágenes satelitales (a través de Google Earth), que permitieron actualizar algunos de los cambios en períodos inter-censales.

En la Tabla 2 se definen los indicadores que componen cada dimensión del ICV y sus fuentes.

Tabla 1. Escala cromática para mapear indicadores, subíndices e índices

\begin{tabular}{|c|c|c|c|}
\hline $\begin{array}{l}\text { Intervalo } \\
\text { numérico }\end{array}$ & $\begin{array}{l}\text { Valor cualitativo del } \\
\text { indicador, subíndice e ICV }\end{array}$ & $\begin{array}{l}\text { Color asociado en los } \\
\text { mapas para indicadores }\end{array}$ & $\begin{array}{c}\text { Color asociado en los } \\
\text { mapas para subín- } \\
\text { dices e índices }\end{array}$ \\
\hline 0 a 0,5 & Muy bajo & & \\
\hline 0,5 a 0,63 & Bajo & & \\
\hline 0,63 a 0,75 & Medio & & \\
\hline 0,75 a 0,87 & Alto & & \\
\hline 0,87 a 1 & Muy alto & & \\
\hline
\end{tabular}

Fuente: elaboración personal 
Tabla 2. Indicadores de cada dimensión del ICV y fuentes utilizadas

\begin{tabular}{|c|c|}
\hline Dimensión & Indicador \\
\hline \multirow{4}{*}{$\begin{array}{l}\text { Dimensión } \\
\text { vivienda }\end{array}$} & $\begin{array}{l}\text { 1. Hacinamiento: porcentaje de personas que, en determinado radio } \\
\text { censal, viven en condición de hacinamiento, es decir en viviendas } \\
\text { donde la cantidad de habitantes por habitación es mayor a } 2 \text { (dos) }\end{array}$ \\
\hline & $\begin{array}{l}\text { 2. Gas por red: porcentaje de personas que, en un radio censal dado, } \\
\text { posee cobertura del servicio de gas por red. }\end{array}$ \\
\hline & $\begin{array}{l}\text { 3. Calidad de la vivienda: Porcentaje de población que, en determinado } \\
\text { radio censal, vive en un hogar de buena calidad (INMAT } 1 \text { o } 2 \text { según } \\
\text { clasificación del INDEC). }\end{array}$ \\
\hline & $\begin{array}{l}\text { 4. Certeza del dominio: Porcentaje de población que, en un radio censal de- } \\
\text { terminado, habita en viviendas de las cuales son propietarios o inquilinos. }\end{array}$ \\
\hline
\end{tabular}

1. Centros de salud: porcentaje de la población que, en un radio censal determinado, vive a una distancia aceptable a un efector de salud. Se adoptó como situación aceptable vivir a 1.000 metros o menos de un Centro de Atención Primaria (CAP) o de un hospital público que tenga dicho servicio.

Dimen-

sión salud

pública

Dimensión

educación ${ }^{a}$
2. Servicios sanitarios básicos: porcentaje de población, a nivel de radio censal, que cuenta de manera simultánea con servicios de agua y cloacas por red en sus viviendas.

3. Basurales a cielo abierto: porcentaje de población que, en un determinado radio censal, está en el área de influencia de un basural a cielo abierto. Para cada tipología de basural se dividió al área de influencia en anillos múltiples a los que se les asignó distintos grados de afectación, de forma de considerar la disminución del efecto a medida que aumenta la distancia a la fuente:

\begin{tabular}{|l|l|l|l|}
\multicolumn{1}{l|}{} & \multicolumn{2}{l}{ Grado de Afectación } \\
\cline { 2 - 4 } & 1 & $1 / 2$ & $1 / 3$ \\
\hline Microbasural (15m3-500m3) & $0-100 \mathrm{~m}$ & $100-200 \mathrm{~m}$ & $200-300 \mathrm{~m}$ \\
\hline Basural (500m3-15000m3) & $0-250 \mathrm{~m}$ & $250-500 \mathrm{~m}$ & $500-750 \mathrm{~m}$ \\
\hline Macrobasural (>15000m3) & $0-500 \mathrm{~m}$ & $500-1000 \mathrm{~m}$ & $1000-1500 \mathrm{~m}$ \\
\hline Depósitos de chatarra & $0-200 \mathrm{~m}$ & $200-400 \mathrm{~m}$ & $400-500 \mathrm{~m}$ \\
\hline
\end{tabular}

A la suma de las tres poblaciones, en un determinado radio censal, la denominaremos "población en el área de influencia de basurales".

1. Años esperados de educación: años de escolarización que un niño, en edad de ingresar a la escuela, puede esperar hasta cumplir los 25 años, si las tasas de matriculación específicas por edad se mantuvieran constantes durante toda su vida.

2. Años promedio de educación: años de educación promedio de la población mayor a 25 años, edad a la que se asume completó su educación formal.
Fuentes

Censo Nacional de Población, Hogares y Viviendas (INDEC, 2010)

SISA del Ministerio de Salud de la Nación; Portal de Geosalud del Ministerio de Salud de la provincia de Buenos Aires; Municipalidades de la cuenca.

Censo Nacional de Población, Hogares y Viviendas (INDEC, 2010), ABSA

ACUMAR

Censo Nacional de Población, Hogares y Viviendas (INDEC, 2010) 
1. Espacios verdes públicos (EVP): porcentaje de población que, en un radio censal determinado, vive en el área de influencia de, al menos, un espacio verde público, apto para la realización de actividades recreativas, deportivas y culturales. Radio de influencia: 500 metros para una plaza o un parque de escala urbana y 5000 metros para un parque de escala metropolitana.

ACUMAR

2. Riesgo por inundación: para el cálculo de este indicador se tomó como insumo la medición antecedente realizada en la Cuenca Matanza Riachuelo por la Facultad de Ingeniería de la UNLP (Ortiz, Carner y Liscia, 2020), la cual buscó determinar los riesgos a las personas causados por inundación. Para ello, dicho estudio utilizó el "Índice de peligrosidad HR", el cual está compuesto por los siguientes elementos: profundidad y la velocidad del agua, y el flujo de escombros. Se consideró asimismo una recurrencia de 10 años. Al respecto, se tomaron los siguientes parámetros para el ICV:

$-\mathrm{HR}>0.75$ tiene un grado de afectación igual a 1

- $0<\mathrm{HR}<0.75$ grado de afectación igual a $1 / 2$

Para cada radio censal se suman estas poblaciones con su grado de afectación y se calcula el porcentaje sobre la población total del radio.

3. Cavas: Porcentaje de población que, en un radio censal determinado, reside en el área de influencia cavas. Se considera que la presencia de cavas afecta en distinto grado a la población según la distancia a la misma:

- Hasta una distancia de $200 \mathrm{~m}$ tiene un coeficiente de afectación igual a 1 (equivalente al 100\% de la población incluida en el círculo).

- En un anillo de radios de 200 a 400 m el coeficiente de afectación es igual a $1 / 2$ (equivalente al $50 \%$ de la población incluida en dicho anillo).

Dimensión entorno - En un anillo de radios de 400 a $600 \mathrm{~m}$ el coeficiente de afectación es igual a $1 / 3$ (equivalente al $33 \%$ de la población incluida en dicho anillo). A la suma de las tres poblaciones, en un determinado radio censal, la denominaremos "población en el área de influencia de cavas".

4. Transporte público: porcentaje de población, en un radio censal determinado, que vive en el área de influencia del recorrido del transporte público de pasajeros (línea de colectivo urbano o interurbano y tren) ${ }^{b}$. Área de influencia:

Facultad de Ingeniería de la UNLP, Laboratorio de $\mathrm{Hi}$ dromecánica

\section{ACUMAR}

- Línea de colectivos urbano o interurbano: 300 metros, medidos en dirección transversal a su recorrido.

- Línea de tren: 1000 metros, medidos desde cada estación de trenes.

5. Industrias: porcentaje de población que, en un radio censal dado, reside en el área de influencia de industrias con impacto ambiental significativo. Se considera que cada tipo de industria afecta en distinto grado a la población según la distancia que tenga a la misma. En resumen: - Industrias de $2^{\circ}$ categoría: hasta una distancia de $100 \mathrm{~m}$ se asume un coeficiente de afectación igual a 1 (equivalente al $100 \%$ de la población); en una distancia de 100 a $300 \mathrm{~m}$ el coeficiente de afectación es igual a $1 / 2$ (equivalente al $50 \%$ de la población) y en una distancia de 300 a $500 \mathrm{~m}$ el coeficiente de afectación es igual a 1/3 (equivalente al $33 \%$ de la población).

- Industrias de $3^{\circ}$ categoría: hasta una distancia de $300 \mathrm{~m}$ se asume Ministerio de Transporte de la Nación un coeficiente de afectación igual a 1 (equivalente al $100 \%$ de la población); en una distancia de 300 a $600 \mathrm{~m}$ el coeficiente de afectación es igual a $1 / 2$ (equivalente al $50 \%$ de la población) y en una distancia de 600 a $1000 \mathrm{~m}$ el coeficiente de afectación es igual a $1 / 3$ (equivalente al $33 \%$ de la población).

a Para el diseño de esta dimensión, se ha seguido la metodología que se usó para adaptar el Índice de Desarrollo Humano (IDH) a nivel municipal en un país latinoamericano. Particularmente, se tomó como referencia una adapta- 
ción realizada en México por parte de un equipo de expertos de Naciones Unidas (PNUD, 2014, 2018). El corte en los 25 años delimita el alcance de los dos indicadores de la dimensión, haciendo que toda la población quede cubierta y no haya superposición. La "tasa esperada de escolarización" es la medida elegida por el IDH para medir el nivel educativo en los menores de 25, mientras que los "años promedio de educación" es un indicador que cubre la educación de parte de los jóvenes y adultos, mayores de 25 años de edad. De esta manera se hace uso de un indicador validado a nivel internacional y pasible de incorporarse al ICV cumpliendo con los requisitos de rigurosidad y parsimonia.

${ }^{b}$ Se tomó este indicador porque se quiso evaluar la existencia o no del servicio de transporte público y no su calidad. Además, sobre esta última cuestión no existía información accesible, que era una de las condiciones para generar los distintos indicadores del ICV

\section{Fuente: elaboración personal}

El origen de los diferentes datos no es exactamente de la misma fecha, fundamentalmente los de población se originan en el año 2010, y el resto provienen de fechas más cercanas a la actual. Esto hace que el ICV sea más preciso para áreas urbanas consolidadas (con pocas variaciones demográficas intercensales) y menos preciso para las nuevas urbanizaciones, formales e informales, que no fueron relevadas por el último censo. Para salvar esta limitación sería conveniente que las autoridades pertinentes lleven un registro detallado de las nuevas urbanizaciones y de sus características.

En la construcción del ICV fue clave tanto la definición de la forma de agregación de los indicadores dentro de cada dimensión y los pesos relativos en las mismas, como la forma de agregación de las dimensiones en el ICV y el peso de cada una en dicho índice.

Respecto a la ponderación de las dimensiones, después de un cuidadoso análisis y debate encarado por el grupo de investigación, se decidió otorgar igual peso a cada dimensión por no existir fundamentos fuertes que permitieran indicar que una dimensión era más importante que otra. De hecho, el proceso mismo de elegir qué dimensiones incluiría el ICV al inicio de la investigación, implicó una profunda reflexión sobre qué temáticas incluir y cuáles no en la arquitectura del instrumento. Finalmente se decidió utilizar (entre un menú de muchas opciones) las cuatro dimensiones que fueron consideradas más importantes para el área bajo estudio, en igual grado de importancia. Todo ello a los fines de ganar parsimonia en el diseño del instrumento y facilidad en su comprensión ${ }^{8}$.

Para otorgar pesos a los indicadores que integran cada subíndice se utilizó el denominado Proceso Analítico Jerárquico (AHP por sus siglas en inglés "Analytic Hiererchy Process"), introducido por Saaty y Wind (1980). En apretada síntesis, el AHP es una técnica que permite evaluar alternativas y ordenarlas en función de su importancia, para así lograr identificar qué alternativas son prioritarias y cuáles menos importantes. Para ello se usan criterios específicos y pertinentes al problema bajo análisis. En el caso del ICV, se usó esta técnica para comparar los indicadores al interior de cada dimensión (por pares) $y$, en función de este proceso, se definió un orden jerarquizado de importancia, que luego permitió asignarles pesos diferenciales. Como puede verse, el punto clave de esta técnica es elegir con cuidado los criterios que permitan hacer las comparaciones y valoraciones. En el caso del ICV, dichos criterios fueron debatidos largamente y decididos al interior del equipo de investigación ${ }^{9}$. Así, este método estructura el proceso de decisión a través de prioridades que llevan a una decisión más eficiente, incorporando aspectos cualitativos y cuantitativos, con el fin de ordenar los componentes en una estructura jerárquica, y así poder asignarle valores numéricos (Pacheco y Contreras, 2008).

8 Esta misma decisión metodológica puede verse en índices internacionales y reconocidos, como el Índice de Desarrollo Humano (IDH) el cual otorga el mismo peso a sus tres dimensiones: salud, economía y educación.

9 Por falta de espacio no podemos desarrollar exhaustivamente el AHP, pero para conocer mejor esta técnica se sugiere ver Saaty y Wind (1980) y Jiménez (2002). 
La Tabla 3 muestra las fórmulas del ICV y de los subíndices resultantes del proceso de diseño.

En cuanto al proceso de agregación, se ha decidido utilizar la media ponderada, siendo un método simple, que evita que se destruya el procedimiento previo realizado. Existen dos formas de utilizar la media ponderada: Media Ponderada Aritmética (MPA) o Media Ponderada Geométrica (MPG). Se probaron ambas fórmulas procurando que el resultado final resulte sensible a los distintos indicadores y que, a la vez, el ICV no resulte 0 en ningún radio censal, ya que se considera que en todos los casos habrá algún factor que incida positivamente en la calidad de vida. Al respecto, se decidió la MPA como forma de agregación en los subíndices (excepto en el vinculado a la dimensión educación, respetando la fórmula propuesta por Naciones Unidas para el IDH), minimizando la posibilidad de que los mismos puedan dar 0 , y se usó la MPG para integrar los subíndices en el ICV ${ }^{10}$.

Tabla 3. Fórmulas de los índices y subíndices del ICV

\begin{tabular}{|c|c|}
\hline Índice o subíndice & Fórmula \\
\hline \multirow[b]{2}{*}{ Índice de calidad de vida } & $I C V=S I V^{(1 / 4)} * S I S P^{(1 / 4)} * S I E d^{(1 / 4)} * S I E n^{(1 / 4)}$ \\
\hline & $\begin{array}{l}\text { SIV: subíndice vivienda; SISP: subíndice salud pública; SIEd: subíndice } \\
\text { educación; SIEn: subíndice entorno }\end{array}$ \\
\hline \multirow{2}{*}{$\begin{array}{l}\text { Subíndice dimen- } \\
\text { sión vivienda }\end{array}$} & $S I V=0,263 * H+0,141 * G R+0,455 * C V+0,141 * C D$ \\
\hline & $\begin{array}{l}\text { H: Hacinamiento; GR: Gas por red; CV: Calidad de la vivienda; CD: } \\
\text { Certeza del dominio }\end{array}$ \\
\hline \multirow[b]{2}{*}{$\begin{array}{l}\text { Subíndice } \\
\text { salud pública }\end{array}$} & $S I S P=0,25 * C S+0,50 * S S B+0,25 * D R$ \\
\hline & $\begin{array}{l}\text { CS: Centros de salud; SSB: Servicios sanitarios básicos; DR: Disposi- } \\
\text { ción residuos }\end{array}$ \\
\hline \multirow{3}{*}{$\begin{array}{l}\text { Subíndice dimensión edu- } \\
\text { cación }\end{array}$} & $S I E d=A E^{0.50} * A P^{0.50}$ \\
\hline & AE: Años esperados de educación para la población menor a 25 años; \\
\hline & AP: Años promedio de educación de la población mayor a 25 años. \\
\hline \multirow{2}{*}{$\begin{array}{l}\text { Subíndice dimen- } \\
\text { sión entorno }\end{array}$} & $S I E n=0,184 * E P V+0,098 * C+0,184 * T P+0,349 * I+0,184 * R I$ \\
\hline & $\begin{array}{l}\text { EPV: Espacios verdes públicos; C: Cavas; TP: Transporte público; I: } \\
\text { Industrias; RI: Riesgo por inundación. }\end{array}$ \\
\hline
\end{tabular}

Fuente: elaboración personal

\section{Resultados}

A través del procesamiento realizado a través de un SIG, según la descripción realizada en el ítem anterior, se obtuvo el mapeo de los indicadores individuales, de los subíndices (asociados a cada dimensión) y del ICV. Por razones de espacio, aquí no se incluyen los mapas de cada uno de los indicadores pero se exponen a continuación aquellos correspondientes a los subíndices (Mapas 1 a 4) y el mapa donde puede verse el resultado del ICV (Mapa 5).

Se puede observar que las dimensiones del ICV tienen un patrón similar de distribución, en el cual los radios censales en mejor situación son los que se encuentran en la cuenca baja y que, asimismo, están fuera del área de riesgo de inundación (Mapas 1 a 4), alejados del curso principal del río. Esto es consecuencia del compor-

10 Si bien el ICV tiene como límites teóricos 0 y 1 , no tiene la posibilidad de alcanzar el 0 ya que nos hemos asegurado que los distintos subíndices que lo integran, por su diseño matemático, sean distintos de 0 . 
tamiento individual de los indicadores que componen cada dimensión, en general de distribución similar a la indicada.

\section{Mapas 1 a 4. Subíndices que integran el ICV}

Mapa 1. Subíndice vivienda

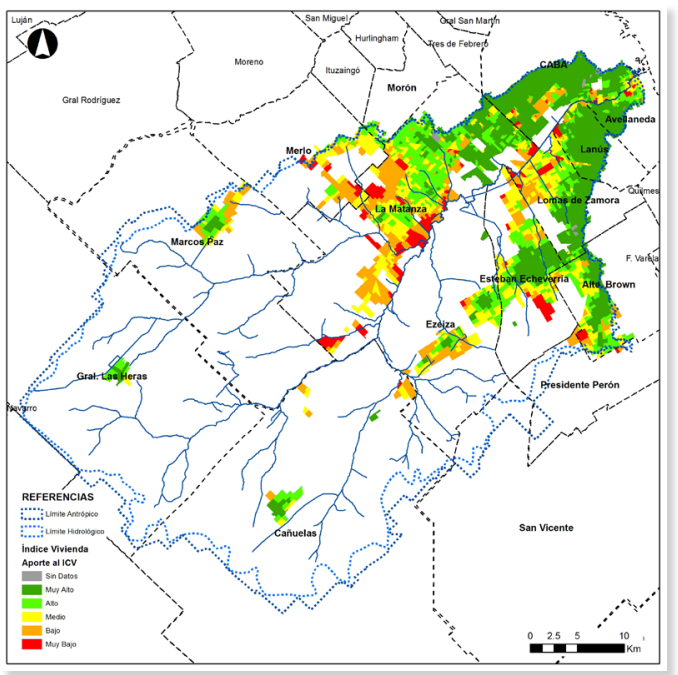

Fuente: elaboración personal con base en Censo Nacional de Población, Hogares y Viviendas (INDEC, 2010)

Mapa 3. Subíndice entorno

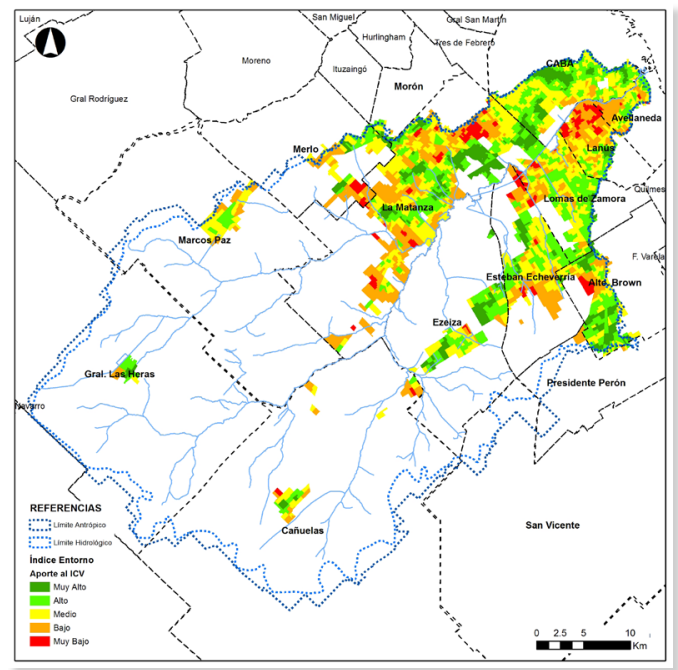

Fuente: elaboración personal con base en ACUMAR y Ortiz et al. (2020); Ministerio de Transporte de la Nación; Organismo Provincial para el Desarrollo Sostenible
Mapa 2. Subíndice educación

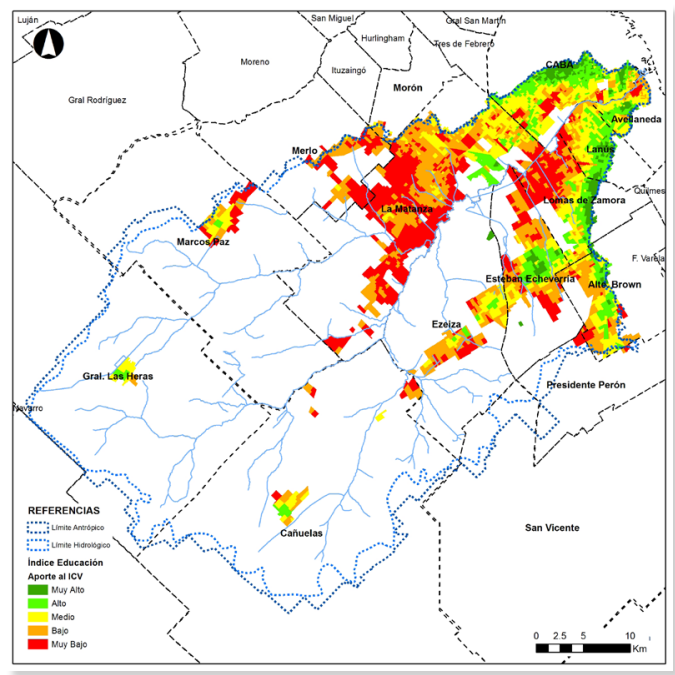

Fuente: elaboración personal con base en Censo Nacional de Población, Hogares y Viviendas (INDEC, 2010)

Mapa 4. Subíndice salud pública

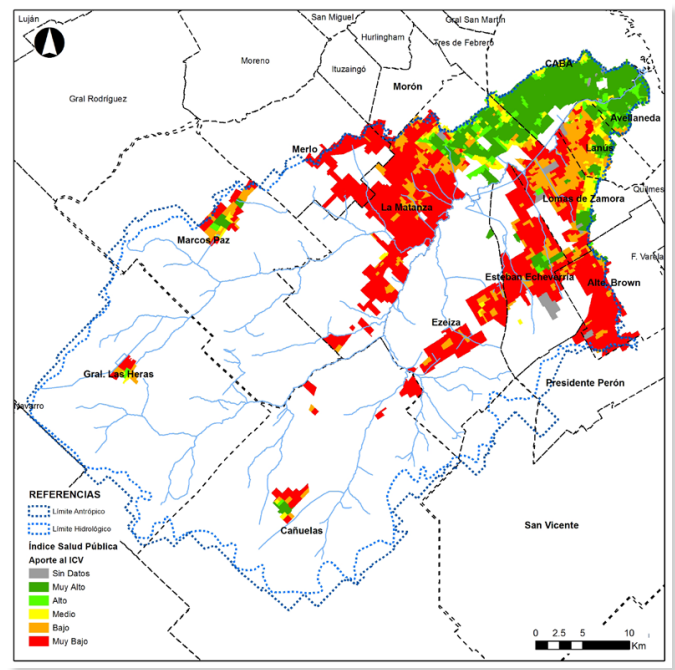

Fuente: elaboración personal con base en SISA del Ministerio de Salud de la Nación; Portal de Geosalud del Ministerio de Salud de la provincia de Buenos Aires; Municipalidades de la cuenca; Censo Nacional de Población, Hogares y Viviendas (INDEC, 2010); ABSA; ACUMAR 


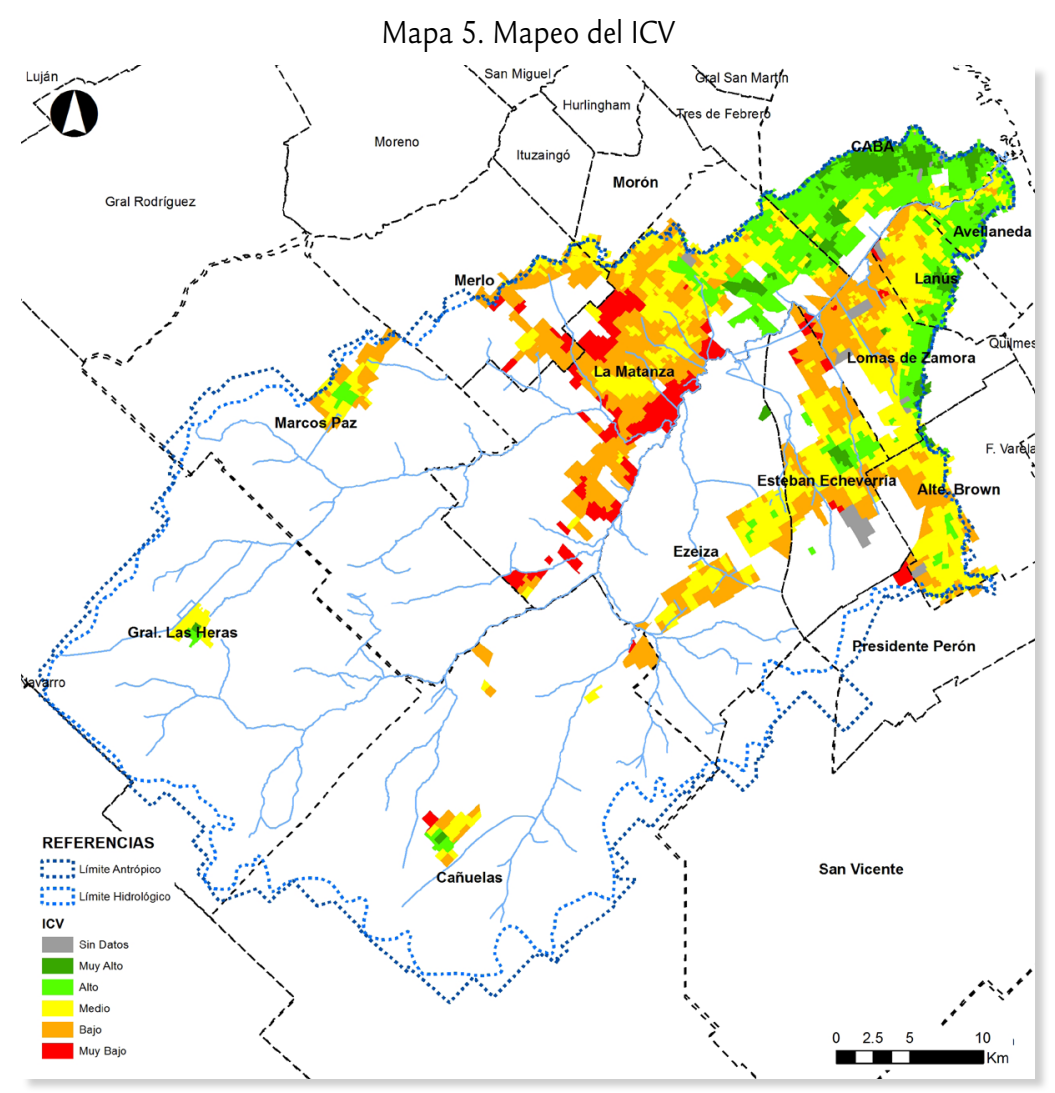

Fuente: elaboración personal con base en Censo Nacional de Población, Hogares y Viviendas (INDEC, 2010); ACUMAR; Ortiz et al. (2020); ISA del Ministerio de Salud de la Nación; Portal de Geosalud del Ministerio de Salud de la provincia de Buenos Aires; Municipalidades de la cuenca; ABSA; Ministerio de Transporte de la Nación; Organismo Provincial para el Desarrollo Sostenible.

Las excepciones más notables se encuentran cuando abrimos el subíndice de la dimensión entorno. Allí puede apreciarse que, contrariamente al patrón general de los otros indicadores, las industrias se hallan ubicadas mayormente en la cuenca baja, influyendo negativamente en la calidad de vida desde un punto de vista ambiental, y las inundaciones tienen influencia en donde se desarrollan, mayormente en la margen derecha de la cuenca baja. Asimismo, las cavas (dimensión entorno) y los basurales (dimensión salud pública) se encuentran mayormente en áreas rurales con lo cual no es muy notable su influencia.

En los Mapas 6 y 7 se muestran los indicadores vinculados a industrias e inundaciones, para visualizar lo indicado en el párrafo anterior.

En el caso de las industrias, hacen un aporte negativo a la salud y calidad de vida a través de acciones tales como: emisiones gaseosas y efluentes industriales, acopio de residuos, ruidos, entrada y salida de camiones y congestión de tránsito. Sin embargo, estas mismas industrias aportan empleo a la población con el consecuente beneficio económico a la comunidad, como se indicó, ello no ha sido evaluado de manera directa en este estudio en función del marco teórico adoptado. 
Mapa 6. Indicador industrias

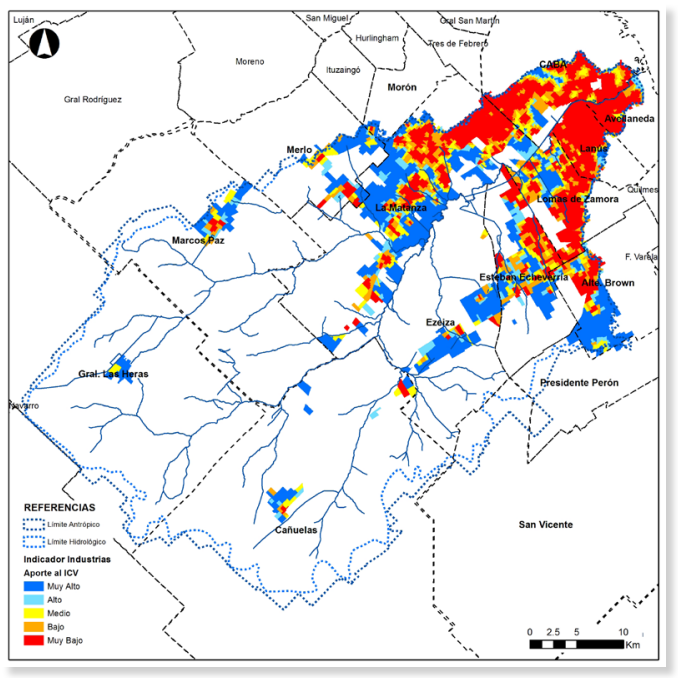

Fuente: elaboración personal con base en Organismo Provincial para el Desarrollo Sostenible y ACUMAR
Mapa 7. Indicador inundaciones

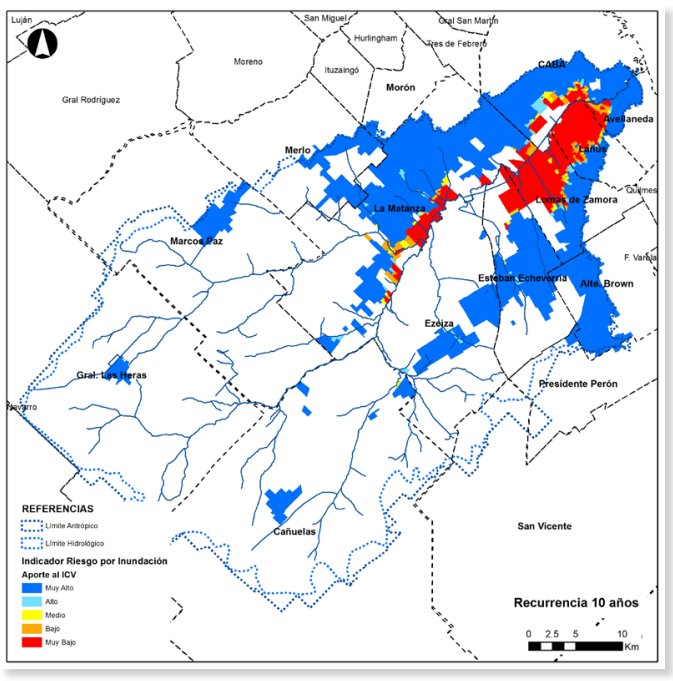

Fuente: elaboraciónpersonal con base en Ortiz et al. (2020)

\section{DISCUSIÓN Y CONCLUSIONES}

Se considera que la presencia de la CABA, ubicada en la margen izquierda de la cuenca baja, con una alta calidad de vida en todo su territorio (ya que es favorecida por el mayor PBI per cápita del país), en conjunto con las características intrínsecas de la cuenca, tienen una influencia significativa en el patrón de distribución del ICV. Asimismo, el emplazamiento histórico de la CABA respondió a la posibilidad de instalar el puerto en las márgenes del Río de la Plata, lo cual hizo que naturalmente parte de su urbanización se ubicara en la parte baja de la Cuenca Matanza Riachuelo, que desemboca en dicho río.

Ese patrón de distribución predominante en la mayor parte de los indicadores se hace notar en el mapa del ICV, con características similares, aunque con matices. Es notable observar que dicho patrón está más vinculado a la ubicación relativa de la población en la cuenca que a la jurisdicción a la que pertenece, siendo CABA una condición de borde determinante para el resto de las jurisdicciones, como se dijo.

La utilización de la cuenca como unidad de análisis parece así una decisión adecuada, al menos en este caso, verificándose al respecto la hipótesis adoptada como parte del planteo inicial para generar el ICV.

Un debate que podría plantearse al respecto, para mejorar la gestión del territorio, sería evaluar la posibilidad de modificar gradualmente los límites político-administrativos de las jurisdicciones haciéndolos coincidir con los límites de cuencas o subcuencas, o al menos considerar a estas últimas como uno de los aspectos determinantes al momento de rever dichos límites. 


\section{AgradeCIMIENTOS}

Se agradece a los técnicos de ACUMAR que han hecho de contraparte en el Convenio Específico $N^{\circ} 1$ con la UNLP a través del cual se realizó la presente investigación, nos referimos a: Mauricio Ferrari, Paula Blua, Ximena Sanchez, Juan Pablo Barabino y Diego Fragas, quienes realizaron aportes valiosos a estudio. Agradecemos al Médico General Daniel Rubén Agüero, que respondió consultas del equipo sobre la dimensión salud. Asimismo, se agradece a quienes participaron del taller final de validación del ICV: además de los mencionados anteriormente, Cecilia Valea, Eugenio Coconier y Álvaro Argüello de ACUMAR, Claudia Natenzon UBA/FLACSO, Nicolás Epele del Ministerio de Economía de la Provincia de Buenos Aires, Silvia Tomás de la Dirección de Estadística de la Prov. de Buenos Aires, Julia Branne, Enrique Angheben y Laura Mordasini de la UNLP.

\section{REFERENCIAS}

Actis Di Pasquale, E. (2008). Bienestar social: un análisis teórico y metodológico como base para la medición de la dinámica histórica en la Argentina. XXI Jornadas de Historia Económica. Caseros, 23 al 28 de septiembre. Recuperado de http://nulan.mdp.edu. ar/808/1/00474.pdf (consulta 10 de febrero de 2015).

Actis Di Pasquale, E. (2015). La elaboración de índices sintéticos de bienestar social. Validación teórica y empírica del método de agregación/ponderación. $12^{\circ}$ Congreso Nacional de Estudios del Trabajo. Buenos Aires 5 al 7 de agosto. Asociación Argentina de Especialistas en Estudios del Trabajo. Recuperado de http://www.aset.org.ar/2015/ ponencias/20_DiPasquale.pdf (consulta 4 de mayo de 2017).

Autoridad de Cuenca Matanza Riachuelo ACUMAR (2016). Plan Integral de Saneamiento Ambiental. Actualización PISA 2016. Hacia una Visión Compartida de la Cuenca. Buenos Aires: Acumar. Recuperado de http://www.acumar.gob.ar/wp-content/uploads/2016/12/ PISA-2016.pdf (consulta 20 de agosto de 2019).

Camargo Mora, M.G. (1999). Calidad de vida y capacidades humanas. Revista Geográfica Venezolana, 40(2), 247-259. Recuperado de http://pdfhumanidades.com/sites/default/ files/apuntes/Camargo\%20Mora.pdf (consulta 13 de julio de 2019).

Castaño, E. (2008). Elementos para una metodología del estudio de la calidad de vida. Revista La Sociología en sus Escenarios, (19), 1-15. Recuperado de http://aprendeenlinea. udea.edu.co/revistas/index.php/ceo/article/view/6812 (consulta 10 de abril de 2017).

Cobb, C.W. (2000). Measurement tools and the quality of life. San Francisco: Redefining Progress. Recuperado de http://citeseerx.ist.psu.edu/viewdoc/download?doi=10.1.1.201.9685\&rep=rep1\&type=pdf (consulta 7 de mayo de 2019).

Discoli, C.; San Juan, G.A.; Martini, I.; Barbero, D.A.; Dicroce, L.; Ferreyro, C.; Viegas, G. y Esparza, J. (2013). Calidad de vida en el sistema urbano: una aproximación teórica y metodológica. La Plata: Universidad Nacional de La Plata. Recuperado de http://sedici.unlp. edu.ar/handle/10915/36292 (consulta 5 de mayo de 2017).

Dourojeanni, A. (1994). Políticas públicas para el desarrollo sustentable: la gestión integrada de cuencas. Santiago de Chile: CEPAL. Recuperado de http://repositorio.cepal.org/handle/11362/19210 (consulta 27 de marzo de 2017).

Dourojeanni, A.; Jouravlev, A. y Chavez, G. (2002). Gestión del Agua a Nivel de Cuencas: teoría 
y práctica. Santiago de Chile: CEPAL. Recuperado de http://repositorio.cepal.org/bitstream/handle/11362/6407/1/S028593_es.pdf (consulta 11 de abril de 2017).

Espinosa Ortiz, F. (2014). Aproximación teórica al concepto de calidad de vida. Entre las condiciones objetivas externas y la evaluación subjetiva de los individuos. Revista de Antropología Experimental, (14), 331-347. Recuperado de http://revistaselectronicas. ujaen.es/index.php/rae/article/view/1801 (consulta 15 de abril de 2015).

Fasciolo, G.; Puebla, P.; Mendoza, V. y Cifuentes, O. (2011). Construcción de indicadores de gestión de cuencas: marco teórico, ejemplos y casos. Documento Síntesis del Taller Indicadores de Gestión de Cuencas. Buenos Aires: Red Argentina de Capacitación y Fortalecimiento en Gestión Integrada de los Recursos Hídricos. Recuperado de: http://www. argcapnet.org.ar/uploads/institucional/materiales/5a303cb66305e.pdf (consulta 15 de abril de 2015).

Gil Ospina, A. (2003). Aproximación conceptual a los términos desarrollo, bienestar y calidad de vida. Revista Páginas, (65), 45-68. Recuperado de https://dialnet.unirioja.es/ servlet/articulo?codigo=4897892 (consulta 2 de agosto de 2020).

Instituto Nacional de Estadística y Censos INDEC (2010). Censo nacional de población, hogares y viviendas 2010: censo del Bicentenario: resultados definitivos. Buenos Aires: Instituto Nacional de Estadística y Censos. Recuperado de: https://www.indec.gob.ar/ftp/cuadros/poblacion/censo2010_tomo1.pdf (consulta 10 de agosto de 2020)

Instituto Nacional de Estadística y Censos INDEC (2015). Definiciones. Unidades Geoestadísticas. Cartografía y códigos geográficos del Sistema Estadístico Nacional. Buenos Aires: Instituto Nacional de Estadística y Censos. Recuperado de https://geoservicios.indec.gov. ar/codgeo/index.php?pagina=definiciones (consulta 10 de enero de 2019).

Instituto Nacional de Estadística y Censos INDEC (2019). Indicadores de condiciones de vida de los hogares en 31 aglomerados urbanos. Primer semestre de 2019. Informes Técnicos, 3(204). Buenos Aires: Instituto Nacional de Estadística y Censos. Recuperado de https://www.indec.gob.ar/uploads/informesdeprensa/eph_indicadores_hogares_01_ 19F640741A45.pdf (consulta 10 de enero de 2019).

Jiménez, J.M. (2002). El proceso Analítico Jerárquico (AHP) Fundamentos, metodología y aplicaciones. RECT@ Revista Electrónica de Comunicaciones y Trabajos de ASEPUMA, 1, 2877. Recuperado de https://users.dcc.uchile.cl/ nbaloian/DSS-DCC/ExplicacionMetodoAHP(ve\%20rpaginas11-16).pdf (consulta 02 de agosto de 2016).

Leva, G. (2005). Indicadores de calidad de vida urbana. Teoría y metodología. Quilmes: Universidad Nacional de Quilmes. Recuperado de http://hm.unq.edu.ar/archivos_hm/ GL_ICVU.pdf (consulta 8 de febrero de 2015).

Ministerio de Planificación Metropolitana (2010). Región Metropolitana de Santiago. Índice de Habitabilidad Comunal 2010. Santiago de Chile: Secretaría Regional Ministerial de Planificación y Coordinación Región Metropolitana. Recuperado de http://www.serplacsantiago.cl/publicaciones/estudios/DOCUMENTO_INDICE_HABITABILIDAD-2010. pdf (consulta 10 de marzo de 2015).

Organización para la Cooperación y el Desarrollo Económicos OECD (2011). Compendium of Key Well-Being Indicators. Paris: Organisation for Economic Co-operation and Development. Recuperado de http://www.oecd.org/std/47917288.pdf (consulta 7 de marzo de 2017).

Ortiz, F.; Carner, J.L. y Liscia, S. (2020). Aplicación de indicadores de riesgo en cuen- 
cas para evaluar medidas estructurales. Aplicación en la Cuenca Matanza- Riachuelo. XXIX Congreso Latinoamericano de Hidráulica. XXVI Congreso Nacional De Hidráulica. Acapulco, octubre de 2020.

Pacheco, J.F. y Contreras, E. (2008). Manual metodológico de evaluación multicriterio para programas y proyectos. Santiago de Chile: CEPAL. Recuperado de https://repositorio.cepal.org/ bitstream/handle/11362/35914/1/manual58_es.pdf (consulta 18 de junio de 2016).

Pena Trapero, B. (2009). La medición del Bienestar Social: una revisión crítica. Estudios de Economía Aplicada, 27(2), 299-324. Recuperado de https://www.redalyc.org/ pdf/301/30117056001.pdf (consulta 12 de diciembre de 2019).

Precedo Ledo, A.; Míguez Iglesias, A. y Orosa González, J. (2012). La calidad de vida de las ciudades gallegas: una aplicación empírica. Boletín de la Asociación de Geógrafos Españoles, (59), 275-300. Recuperado de https://bage.age-geografia.es/ojs/index.php/ bage/article/view/1458/1379 (consulta 3 de mayo de 2017).

Prieto, M.B. (2013). Diferenciación socio-espacial y condiciones de vida en Bahía Blanca (1991-2001). Hologramática, 1(17), 43-72. Recuperado de http://cienciared.com.ar/ra/ usr/3/1442/hologramatica_n18v1pp43_72.pdf (consulta 4 de abril de 2017).

Programa de las Naciones Unidas para el Desarrollo PNUD (2014). Índice de desarrollo humano municipal en México: nueva metodología. México: Programa de las Naciones Unidas para el Desarrollo. Recuperado de https://www.undp.org/content/dam/mexico/ docs/Publicaciones/PublicacionesReduccionPobreza/InformesDesarrolloHumano/ UNDP-MX-PovRed-IDHmunicipalMexico-032014.pdf (consulta 19 de mayo de 2020).

Programa de las Naciones Unidas para el Desarrollo PNUD (2018). Índice e indicadores de desarrollo humano. Actualización estadística 2018. Nueva York: Programa de las Naciones Unidas para el Desarrollo. Recuperado de http://hdr.undp.org/sites/default/files/2018_human_development_statistical_update_es.pdf(consulta 19 de mayo de 2020).

Saaty, T. y Wind, Y. (1980). Marketing applications of the analytic hierarchy process. Management science, 26(7), 641-658.

United Nations (2004). Urban Indicators Guidelines. Monitoring the Habitat Agenda and the Millennium Development Goals. Nairobi: United Nations Human Settlements Programme. Recuperado de https://unhabitat.org/sites/default/files/download-manager-files/ Urban\%20Indicators.pdf (consulta 10 de agosto de 2020).

Velázquez, G.; Longhi, F.; Mikkelsen, C. y Celemín, J.P. (2013). Estudios sobre geografía y calidad de vida en la Argentina. Cinco décadas de aportes bibliográficos. Hologramática, 19(1), pp. 77-105. Recuperado de https://dialnet.unirioja.es/servlet/articulo?codigo=5869599 (consulta 21 de agosto de 2020).

Zulaica, L. y Celemín, J.P. (2008). Análisis territorial de las condiciones de habitabilidad en el periurbano de la ciudad de Mar del Plata (Argentina), a partir de la construcción de un índice y de la aplicación de métodos de asociación espacial. Revista de Geografía Norte Grande, (41), 129-146. Recuperado de http://www.scielo.cl/scielo.php?script=sci_arttext\&pid=S0718-34022008000300007 (consulta 19 de marzo de 2017).

\section{OTRAS FUENTES}

Sistema Argentino de Información Jurídica SAIJ (2008). Mendoza, Beatriz Silvia y otros c/ Estado Nacional y otros s/ daños y perjuicios (daños derivados de la contaminación ambiental del Río Matanza-Riachuelo). Ministerio de Justicia y Derechos Humanos Argentina. Recuperado de 
http://www.saij.gob.ar/corte-suprema-justicia-nacion-federal-ciudad-autonoma-buenos-aires-mendoza-beatriz-silvia-otros-estado-nacional-otros-danos-perjuicios-danos-derivados-contaminacion-ambiental-rio-matanza-riachuelo-fa08000047-2008-07 -08/123456789-740-0008-0ots-eupmocsollaf (consulta el 19 de agosto de 2020).

Sistema Argentino de Información Jurídica SAIJ (2013a). Ley 14.449 de Acceso Justo al Hábitat. Normas de la Prov. de Buenos Aires. Recuperado de http://www.saij.gob. ar/14449-local-buenos-aires-promocion-derecho-vivienda-habitat-digno-sustentable-creacion-programa-lotes-servicios-finalidad-facilitar-acceso-al-suelo-urbanizado-familias-bonaerenses-Ipb0014449-2012-11-29/123456789-0abc-defg-944-4100b vorpyel?q=\%28numero-norma\%3A14449\%20\%29\&o=0\&f=Total\%7CTipo\%20de\%20 Documento/Legislaci\%F3n\%7CFecha\%7COrganismo\%7CPublicaci\%F3n\%7CTema\%7CEstado\%20de\%20Vigencia\%7CAutor\%7CJurisdicci\%F3n/Local/Buenos\%20Aires\&t=1 (consulta el 18 de agosto de 2020).

Sistema Argentino de Información Jurídica SAIJ (2013b). Ley Provincial N 14.782. Reconocer el acceso al agua potable y al saneamiento como un derecho humano esencial para la vida. Recuperado de http://www.saij.gob.ar/LPB0014782 (consulta el 22 de agosto de 2020)

María Laura Henry es Doctora en Ciencias Sociales por la Universidad de Buenos Aires (UBA). Magister en Ciencias Sociales por la Universidad de Buenos Aires (UBA). Licenciada en Sociología por la Universidad Nacional de La Plata (UNLP). Profesora-investigadora de la Facultad de Humanidades y Ciencias de la Educación de la UNLP. Acredita trabajos investigación, de transferencia y publicaciones sobre las temáticas de: condiciones y medio ambiente de trabajo; sociología del trabajo; calidad de vida y metodología de la investigación. Facultad de Humanidades y Ciencias de la Educación. Universidad Nacional de La Plata. Calle 51 e/ 124 y 125, (1925) Ensenada, Buenos Aires, Argentina, mlaurahenry@gmail.com, ORCID https://orcid.org/0000-0002-2869-8506

Marcos Cipponeri es Ingeniero Hidráulico (1991) Facultad de Ingeniería, Universidad Nacional de La Plata. Ingeniero Civil (1992) Facultad de Ingeniería, Universidad Nacional de La Plata. Es profesor ordinario con dedicación exclusiva en la Facultad de Ingeniería de la UNLP. Entre sus principales actividades se encuentran: a) profesor de las asignaturas Gestión Ambiental e Introducción a la Gestión Integrada de Cuencas Hidrográficas, b) Coordinador de la Unidad de Investigación, Desarrollo, Extensión y transferencia Gestión Ambiental, c) profesor de la Maestrías en Ecohidrología, en Arquitectura y Hábitat Sustentable y en Ingeniería Vial, todas de la UNLP, d) Presidente de la Red Argentina de Capacitación y Fortalecimiento en Gestión Integrada de los Recursos Hídricos. Sus temas de investigación están vinculados a la gestión integrada de cuencas hidrográficas y a los estudios ambientales vinculados a las grandes obras de infraestructura. Facultad de Ingeniería. Universidad Nacional de La Plata. Calle 47 N²00, (1900) La Plata, Buenos Aires, Argentina, mcipponeri@ing.unlp.edu.ar, ORCID https://orcid.org/0000-0002-9533-4366

Valentina Bonifacio Magister en Economía (2016) Facultad de Ciencias Económicas, Universidad Nacional de La Plata. Licenciada en Economía (2014) Facultad de Ciencias Económicas, Universidad Nacional de La Plata. Trabaja en el Fondo Monetario Internacional como Analista de Investigación en temas relacionados con macroeconomía global. Anteriormente, trabajó en El Banco Mundial en Washington en temas de pobreza y desigualdad del ingreso para El Salvador y Costa Rica. Su tesis de posgrado se basa en temas sociales, específicamente, en la dinámica de la pobreza en Argentina. Su trabajo 
en Argentina estuvo focalizado en la consultoría externa para proyectos de investigación en temas socioeconómicos en la Universidad Nacional de La Plata, tanto para la Facultad de Ciencias Económicas como para Facultad de Ingeniería. Fondo Monetario Internacional. 1900 Pennsylvania Ave, Washington, Distrito de Columbia, Estados Unidos, valentina.bonifacio28@gmail.com, ORCID https://orcid.org/0000-0002-6811-3437

Mónica Laura Salvioli es Licenciada en Biología con orientación Ecología. Especialista en Ambiente y Patología Ambiental. Docente con dedicación exclusiva. Facultad de Ingeniería, UNLP. Cátedra Gestión Ambiental. Actividades docentes de posgrado. Coordinación/ participación en el desarrollo de estudios ambientales aplicados a planes, programas y proyectos, que permitan analizar y lograr la compatibilidad ambiental de los mismos. Énfasis en el análisis ambiental de grandes obras de infraestructura: presas multipropósito, acueductos, captación, tratamiento y conducción de agua potable y plantas depuradoras de efluentes cloacales, entre otros. Participación en estudios de gestión integrada de recursos hídricos. Investigación y desarrollo de índices de calidad ambiental. Se destaca el desarrollo de índices de calidad de vida y de calidad de agua para la cuenca hídrica Matanza Riachuelo, provincia de Buenos Aires. Universidad Nacional de La Plata. Facultad de Ingeniería. UIDET Gestión Ambiental. Calle 47 N² 200, (1900) La Plata, Buenos Aires, Argentina, msalvioli@ing.unlp.edu.ar, ORCID https://orcid.org/0000-0002-4104-1701

Guillermo Larrivey es Ingeniero Hidráulico e Ingeniero Civil por la Universidad Nacional de La Plata (UNLP). Se especializó en manejo de programas de Diseño Asistido por Computadora (CAD) y Sistemas de Información Geográfica (SIG). Desde el año 1997 forma parte de la UIDET Gestión Ambiental, Facultad de Ingeniería (UNLP), que trabaja en el desarrollo de planes, programas y proyectos que incluyan variables ambientales. Dentro de esta Unidad ha participado en estudios ambientales de grandes obras de infraestructura, en estudios de gestión integrada de recursos hídricos y en el desarrollo de índices de calidad ambiental. En el ámbito privado se ha desempeñado en consultoras que trabajan en el área de la ingeniería, el urbanismo y el desarrollo territorial. Unidad de Investigación, Desarrollo, Extensión y Transferencia UIDET. Facultad de Ingeniería. Universidad Nacional de La Plata. Calle 47 № 200, (1900) La Plata, Buenos Aires, Argentina, glarrivey@ing.unlp.edu.ar, ORCID https://orcid.org/0000-0002-3481-3152

Verónica Guerrero Borges es Magister en Ciencias del Territorio, Facultad de Arquitectura y Urbanismo, Universidad Nacional de La Plata. Licenciada en Biología, Facultad de Ciencias Naturales y Museo, Universidad Nacional de La Plata. Se desempeña actualmente como Docente en Gestión Ambiental en la Facultad de Ingeniería (ing. Civil e Hidráulica) y Estadística aplicada a las Ciencias Naturales, en la Facultad de Ciencias Naturales y Museo de la UNLP. Sus estudios de postgrado se orientaron a la implementación de Sistemas de Información Geográfica, análisis de sensores remotos aplicados a las ciencias ambientales y la gestión del recurso hídrico, al estudio de impacto ambiental de obras de infraestructura, ordenamiento territorial y análisis estadístico. Presenta más de 20 publicaciones en revistas y libros tanto nacionales como internacionales en su mayoría en el área evaluación de impacto de grandes obras hidráulicas aplicando modelos SIG, ha participado como expositor en más de 40 congresos nacionales e internacionales y realizado más de 20 cursos de perfeccionamiento en los últimos 10 años en el área de la modelaciones espaciales aplicados a los recursos hídricos. Facultad de Ciencias Naturales y Museo. Facultad de Ingeniería. Universidad Nacional de La Plata. Av. 1 750, (1900) La Plata, Buenos Aires, Argentina, vgborges@gmail.com, ORCID https://orcid.org/0000-0002-0231-9918 